\title{
Active Matter that Mimics Turbulence in Space and Time
}

\author{
Despite being driven by a different process, a system of self-propelling \\ particles can evolve over time in a similar way to a turbulent fluid.
}

By Erika K. Carlson

S ystems of self-propelling particles-whether artificial devices or bacteria in suspension-tend to create patterns in their surrounding media that are reminiscent of turbulent flows. Researchers have previously noted that particles in turbulent fluids, and active swimmers in nonturbulent fluids, produce similar arrangements in space. Now, Mickaël Bourgoin of the École Normale Supérieure in Lyon, France, and colleagues have observed an active-matter system whose behavior mirrors the way fluid turbulence evolves over time as well.

The researchers studied a system of camphor-filled hydrogel disks floating on water. As camphor spread from the disks, it changed the water's surface tension, creating so-called Marangoni gradients that propelled the disks along. The team found, as in past studies, that the spatial organization of this system-meaning the distances and velocity differences between the disks-was statistically very similar to that of nonpropelled particles floating on a turbulent fluid. In a

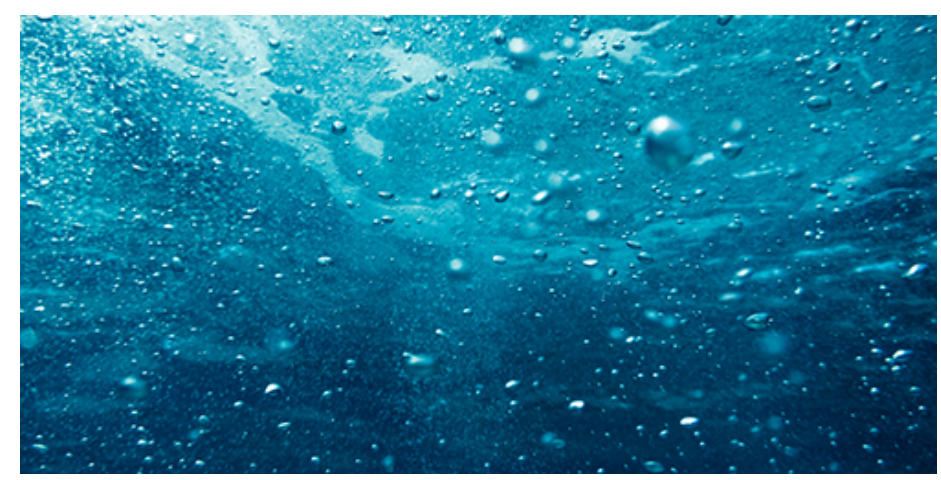

Credit: borchee/iStock/Getty Images complementary approach, the researchers tracked the motions of the disks over time and calculated the dynamics of a fluid that would yield the same statistical evolution had the disks been passive "tracer" particles carried by the fluid. They found that the way the patterns changed over time was consistent with what tracer particles would experience in a turbulent flow. This didn't have to be the case-spatial organization and temporal dynamics are separate phenomena that don't have to be linked via turbulence.

Though active-matter systems and particles driven by turbulent fluids reflect different underlying phenomena, the researchers say that comparing their behavior could lead to new insights. For example, examining the differences that do exist between the two systems' similar behaviors could further our understanding of how turbulent phenomena arise.

This research is published in Physical Review X.

Erika K. Carlson is a Corresponding Editor for Physics based in Brooklyn, New York. 